\title{
Decay rates for second-order linear evolution problems with fractional laplacian operators
}

\author{
Cleverson Roberto da Luz' ${ }^{(\mathbb{D}}$, Maíra Fernandes Gauer Palma'(i) \\ ' Universidade Federal de Santa Catarina, Santa Catarina, SC, Brasil
}

\section{ABSTRACT}

In this work we study the asymptotic behavior of solutions for a general linear second-order evolution differential equation in time with fractional Laplace operators in $\mathbb{R}^{n}$ We obtain improved decay estimates with less demand on the initial data when compared to previous results in the literature. In certain cases, we observe that the dissipative structure of the equation is of regularity-loss type. Due to that special structure, to get decay estimates in high frequency region in the Fourier space it is necessary to impose additional regularity on the initial data to obtain the same decay estimates as in low frequency region. The results obtained in this work can be applied to several initial value problems associated to second-order equations, as for example, wave equation, plate equation, IBq, among others.

Keywords: Asymptotic behavior; fractional Laplace operator; Fourier space; second-order equations

\section{INTRODUCTION}

We consider the following Cauchy problem with fractional Laplace operators in $\mathbb{R}^{n}$ :

$$
v_{t t}(t, x)+(-\Delta)^{\delta} v_{t t}(t, x)+(-\Delta)^{\alpha} v(t, x)+(-\Delta)^{\theta} v_{t}(t, x)=0, t \geq 0, x \in \mathbb{R}^{n}
$$

with initial data

$$
v(0, x)=v_{0}(x), v_{t}(0, x)=v_{1}(x)
$$


where $\delta, \alpha$ and $\theta$ are real numbers with $\delta, \alpha \geq 0$ and $\theta \in[0, \alpha]$. The fractional power operator $(-\Delta)^{\theta}: H^{2 \theta}\left(\mathbb{R}^{n}\right) \rightarrow L^{2}\left(\mathbb{R}^{n}\right)(\theta \geq 0)$ is defined by

$$
(-\Delta)^{\theta} v(x):=\mathcal{F}^{-1}\left(|\xi|^{2 \theta} \hat{v}(\xi)\right)(x), \quad v \in H^{2 \theta}\left(\mathbb{R}^{n}\right), x \in \mathbb{R}^{n},
$$

where $\mathcal{F}$ denotes the usual Fourier transform in $L^{2}\left(\mathbb{R}^{n}\right)$ with respect to the $\mathrm{x}$ variable, $\hat{v}=\mathcal{F}(v), H^{s}=H^{s}\left(\mathbb{R}^{n}\right)$ denotes the usual Sobolev space of $L^{2}$ functions equipped with the norm $\|\cdot\|_{H^{s}}$ and $|\cdot|$ denotes the usual norm in $\mathbb{R}^{n}$. For simplicity of notations, in particular, we use in all text $\|\cdot\|$ instead of $\|\cdot\|_{L^{2}}$. The operator $(-\Delta)^{\theta}$ is nonnegative and self-adjoint in $L^{2}\left(\mathbb{R}^{n}\right)$.

The total energy $E_{v}(t)$ associated to the solution $v(t)$ of equation (1) is defined by

$$
E_{v}(t)=\frac{1}{2}\left\{\left\|v_{t}(t)\right\|^{2}+\left\|(-\Delta)^{\frac{\delta}{2}} v_{t}(t)\right\|^{2}+\left\|(-\Delta)^{\frac{\alpha}{2}} v(t)\right\|^{2}\right\}
$$

In order to obtain estimates for the problem (1) - (2) we work with the corresponding problem in the Fourier space. Applying the Fourier transform with respect to the variable $x$, we obtain

$$
\left\{\begin{array}{c}
\left(1+|\xi|^{2 \delta}\right) \hat{v}_{t t}(t, \xi)+|\xi|^{2 \theta} \hat{v}_{t}(t, \xi)+|\xi|^{2 \alpha} \hat{v}(t, \xi)=0, t \geq 0, \xi \in \mathbb{R}^{n} \\
\hat{v}(0, \xi)=\hat{v}_{0}(\xi), \hat{v}_{t}(0, \xi)=\hat{v}_{1}(\xi), \xi \in \mathbb{R}^{n}
\end{array}\right.
$$

The eigenvalues of the problem (4) have nonpositive real part, and they are given by

$$
\lambda_{ \pm}=\frac{|\xi|^{2 \theta}}{2\left(1+|\xi|^{2 \delta}\right)}\left(-1 \pm \sqrt{1-4|\xi|^{2(\alpha-2 \theta)}\left(1+|\xi|^{2 \delta}\right)}\right) .
$$

The solution of (4) can be written as

$$
\hat{v}(t, \xi)=\widehat{K}_{0}(t, \xi) \hat{v}_{0}(\xi)+\widehat{K}_{1}(t, \xi) \hat{v}_{1}(\xi)
$$

where 


$$
\widehat{K}_{0}(t, \xi)=\frac{\lambda_{+} e^{\lambda_{-} t_{-} \lambda_{-} e^{\lambda_{+} t}}}{\lambda_{+}-\lambda_{-}} \text {and } \widehat{K}_{1}(t, \xi)=\frac{e^{\lambda_{+} t_{-}} e^{\lambda_{-} t}}{\lambda_{+}-\lambda_{-}}
$$

Therefore

$$
v(t, x)=k K_{0}(t, x) * v_{0}(x)+k K_{1}(t, x) * v_{1}(x),
$$

with $\mathrm{k}$ a positive constant such that $\hat{f} \cdot \hat{g}=k(f * g)$.

For $\varepsilon>0$, we denote by $E_{0}(t, x)$ and $E_{\infty}(t, x)$ the solution of (1) localized to low and high frequencies, that is,

$$
\begin{aligned}
& E_{0}=E_{0}(t, x)(v)=\mathcal{F}^{-1}(\chi(\xi) \hat{v}(t, \xi)), \\
& E_{\infty}=E_{\infty}(t, x)(v)=\mathcal{F}^{-1}((1-\chi(\xi)) \hat{v}(t, \xi)),
\end{aligned}
$$

where $\chi(\xi)$ is the characteristic function of $\left\{\xi \in \mathbb{R}^{n} /|\xi|<\varepsilon\right\}$. To estimate the $x$ derivatives norms of $v$ and $v_{t}$ it is sufficient to estimate the derivatives norms of $E_{0}, \partial_{t} E_{0}, E_{\infty}$ and $\partial_{t} E_{\infty}$.

If $\theta<\delta$, we observe that the decay structure of (1) is of regularity-loss type which is characterized by the structure of the eigenvalues associated to the problem. The regularity-loss property ceases to occur in the case when $\theta=\delta$. Due to that special structure, to get decay estimates in the high frequency region in the Fourier space it is necessary to impose additional regularity on the initial data to obtain the same decay estimates as in the low frequency region. If $\delta \leq \theta$ this effect does not appear, since the solution decays exponentially in the high frequency zone of the Fourier space (see Proposition 3.1). Such decay property of the regularity-loss type was also investigated for the dissipative Timoshenko system by Ide and Kawashima (2008), the plate equation under rotational inertia effects in $\mathbb{R}^{n}$ by Sugitani and Kawashima (2010); Charão et al. (2013a); D'Abbicco et al. (2016) and a hyperbolic-elliptic system of a radiating gas model by Kubo and Kawashima (2009).

The case $\theta>\alpha$ is called super damping and was studied by Charão et al. (2020). Due to the presence of strong damping, some different techniques must be used. This case was not considered in this work. 
Estimates for the solution to the wave equation with structural damping

$$
u_{t t}(t, x)-\Delta u(t, x)+2 a(-\Delta)^{\theta} u_{t}(t, x)=0, t \geq 0, x \in \mathbb{R}^{n},
$$

have been derived in Ikehata and Natsume (2012); Charão et al. (2013b); D'Abbicco and Reissig (2014); D'Abbicco and Ebert (2014); Karch (2000), where as the case of time-dependent damping coefficients is considered in Wirth (2012); Lu and Reissig (2009). In D'Abbicco and Ebert (2014), the authors decompose the solution to (8) into two parts, $u=u^{+}+u^{-}$, each one related to one of the two characteristics roots associated to (8). The asymptotic behavior of the Fourier transforms of each part hints to two different diffusion phenomena. This type of problem has been extensively studied in the mathematical and physical literature (see, for instance, Biler et al. (2001); Córdoba and Córdoba (2004); Fino and Karch (2010); Vázquez (2010)). Karch (2000) studied the large time behavior of solutions to the initial value problem (1) with $\delta=0,0 \leq 2 \theta<\alpha$ and a non-linear term $F\left(x, t, u, u_{t}, \nabla \mathrm{u}\right)$. In the cited paper, an analysis of the solution formula of the linear problem leads to the conclusion that they behave, as $t \rightarrow \infty$, like solutions of a similar diffusion equation to the problem in D'Abbicco and Ebert (2014).

Next we mention some important previous works which are related with this paper. Sugitani and Kawashima (2010) studied a semilinear dissipative plate equation with rotational inertia effects and a frictional dissipation. To the linear problem, they used the explicit solution and equivalences for eigenvalues. To the semilinear problem, they introduce a Banach space $X$ defined by the norm

$$
\|u\|_{X}=\sum_{\sigma_{0}(k) \leq s+1} \sup _{t \geq 0}(1+t)^{\frac{k}{4}}\left\|\partial_{x}^{k} u(t)\right\|_{H^{s+1-\sigma_{0}(k)}}
$$

where $\sigma_{0}(k)=k+\left[\frac{k+1}{2}\right]$ and $s \geq \sigma_{0}(k)-1$. They proved that the problem is solved globally in time in the above function space and found optimal decay estimates of 
solutions under the additional regularity assumption on the initial data $u_{0} \in H^{s+1}$ and $u_{1} \in H^{s}$, for s large enough.

D'Abbicco and Reissig (2014) studied the semilinear structural damped wave equation. They obtained optimal decay to the norm of solution, to the energy terms and determined the influence of fractionary dissipation on the critical exponent. The method used for the linear problem is similar to the method applied in Sugitani and Kawashima (2010) (see also Wirth (2012)).

Based on the energy method in Fourier space, introduced by Umeda et al. (1984), several works (see Dharmawardane et al. (2010); Ikehata and Natsume (2012); Ikehata et al. (2013)) showed decay estimates to some evolution equations in $\mathbb{R}^{n}$. Charão et al. $(2013 \mathrm{~b}, \mathrm{a})$ introduced a new method to get decay estimates supported on the energy method in Fourier space combined with the HarauxKomornik inequality, the monotonicity of the energy density in the Fourier space and the property of power singularities less than $n$ are integrable around the origin of $\mathbb{R}^{n}$. They obtained in Charão et al. (2013b) almost optimal decay for wave equation with a fractional damping and in Charão et al. (2013a) for the plate equation with rotational inertia effects and fractional damping. In da Luz et al. (2015) they extended these results to an abstract problem of second order differential equation. The decay rate $E(t)=O\left(t^{-k}\right)$ is almost optimal means that

$$
E(t)=O\left(t^{-k+\varepsilon}\right) \quad(t \rightarrow+\infty)
$$

for any $\varepsilon>0$.

In this work we study a more general equation when compared to the problems (or at least to the associated linear problem) studied in Charão et al. (2013b,a); Ikehata and Natsume (2012); D'Abbicco and Ebert (2014); D'Abbicco and Reissig (2014); Karch (2000); Sugitani and Kawashima (2010); Polat and Ertas (2009); Wang and Xu $(2013,2012)$. The main objective of this work is to improve the results obtained in da Luz et al. (2015). Due to the method used in da Luz et al. (2015), the authors proved almost optimal decay rates only for the total energy of 
order $\alpha$ of the problem, that is, the method does not allow to obtain optimal decay rates for the total energy, as well as does not allows to estimate each term of the energy separately. However, as can be seen in Section 4 of this work, the rates can vary for each term of the energy. In this paper we obtain sharp decay rates for $\left\|\partial_{x}^{\gamma_{1}} v(t)\right\|,\left\|\partial_{x}^{\gamma_{2}} v_{t}(t)\right\|$, with $\gamma_{1,} \gamma_{2} \in \mathbb{N}^{n}$, and the corresponding regularity of the initial data. The regularity of the initial data assumed at this work is not optimal, a more detailed study on this topic is an open problem.

To obtain the desired decay rates, we study low and high frequency regions separately. The next section will deal low frequency, using the ideas presented in D'Abbicco and Reissig (2014) and Sugitani and Kawashima (2010), i.e., we consider the explicit solution of the problem and estimate the eigenvalues. It will be necessary to separate in two cases: real eigenvalues $(\alpha>2 \theta)$ and complex eigenvalues $(\alpha \leq 2 \theta)$.

In the third section we study the problem in high frequency zone through a redesign of the energy method in Fourier space introduced by Charão et al. $(2013 b, a)$. In this section we find the additional regularity required in the in itial data (if $\theta<\delta$ ) to obtain desired decay rates.

In the last section we exhibit and prove the main theorems, combining the results of Sections 2 and 3 . In addition, some important applications are presented, finding sharp decay rates for the norm of solution and for the terms of the energy associated with the wave equation with fractional damping, plate equation with rotational inertia effects and fractional damping and a Boussinesq equation with fractional damping.

The method may further be applied to various other evolution equations in $\mathbb{R}^{n}$ with constant coefficients. Moreover, we can add terms of the type $(-\Delta)^{\delta_{1}} v_{t t},(-\Delta)^{\theta_{1}} v_{t},(-\Delta)^{\alpha_{1}} v$ in equation (1) and get decay rates to the solution of the new problem from the results obtained for the problem (1)-(2). This is possible because, for example, by adding the term $(-\Delta)^{\theta_{1}} v_{t}$ we obtain 


$$
|\xi|^{2 \theta}+|\xi|^{2 \theta_{1}}
$$

as coefficient of the term $\hat{v}_{t}$ at the equation in Fourier space. But this coefficient is equivalent to $|\xi|^{2 \theta_{0}}$, where $\theta_{0}=\min \left\{\theta, \theta_{1}\right\}$ in low frequency, and $\theta_{0}=\max \left\{\theta, \theta_{1}\right\}$ in high frequency. The same occurs when we add terms of the type $(-\Delta)^{\delta_{1}} v_{t t},(-\Delta)^{\alpha_{1}} v$ in the equation (1). It means that using the method presented in this work, it is also possible to obtain estimates for this new problem. In order not to make this a long work, we're going to consider only a simple example in order to illustrate this case (see Subsection 4.2.3).

Note that in this work we do not discuss the existence and uniqueness of solutions because they can be obtained via theory of semigroups.

\section{LOW FREQUENCY REGION: $|\xi|<\varepsilon$}

In the following we use the notation $f \approx g$ if there exist two constants $C_{1}, C_{2}>0$ such that $C_{1} g \leq f \leq C_{2} g$. If the inequality is one-sided, namely, if $f \leq C_{2} g$ (resp. $f \geq C_{1} g$ ), then we write $f \lesssim g$ (resp. $g \lesssim f$ ). Furthermore, we use the symbol $\gamma$ to denote a multi-index with non-negative entries.

We observe that

$$
\begin{gathered}
\left\|\partial_{t}^{j} \partial_{x}^{\gamma} E_{0}(t)\right\|^{2}=\int_{\mathbb{R}^{n}}\left|\partial_{t}^{j} \hat{v}(t)\right|^{2}|\xi|^{2|\gamma|} \chi(\xi)^{2} d \xi \\
\lesssim\left\|\hat{v}_{0}\right\|_{L^{\infty}}^{2} \int_{\mathbb{R}^{n}}\left|\partial_{t}^{j} \widehat{K}_{0}(t)\right|^{2}|\xi|^{2|\gamma|} \chi(\xi)^{2} d \xi+\left\|\hat{v}_{1}\right\|_{L^{\infty}}^{2} \int_{\mathbb{R}^{n}}\left|\partial_{t}^{j} \widehat{K}_{1}(t)\right|^{2}|\xi|^{2|\gamma|} \chi(\xi)^{2} d \xi
\end{gathered}
$$

with $E_{0}$ defined in (6) and $\widehat{K}_{0}, \widehat{K}_{1}$ defined in (5). Setting $I_{0}$ and $I_{1}$ by integrals

$$
I_{0}^{2}(j,|\gamma|):=\int_{\mathbb{R}^{n}} \frac{\mid \partial_{t}^{j}\left(\lambda_{+} e^{\left.\lambda_{-} t_{-} \lambda_{-} e^{\lambda_{+} t}\right)\left.\right|^{2}}\right.}{\left|\lambda_{+}-\lambda_{-}\right|^{2}}|\xi|^{2|\gamma|} \chi(\xi)^{2} d \xi
$$

and 


$$
I_{1}^{2}(j,|\gamma|):=\int_{\mathbb{R}^{n}} \frac{\mid \partial_{t}^{j}\left(e^{\left.\lambda_{+} t_{-} e^{\lambda-t}\right)\left.\right|^{2}}\right.}{\left|\lambda_{+}-\lambda_{-}\right|^{2}}|\xi|^{2|\gamma|} \chi(\xi)^{2} d \xi
$$

it follows from (5) that

$$
\left\|\partial_{t}^{j} \partial_{x}^{\gamma} E_{0}(t)\right\|^{2} \lesssim\left\|v_{0}\right\|_{L^{1}}^{2} I_{0}^{2}(j,|\gamma|)+\left\|v_{1}\right\|_{L^{1}}^{2} I_{1}^{2}(j,|\gamma|)
$$

To obtain the main results of this section, we estimate each one of the integrals $I_{0}$ and $I_{1}$. To do this, we study separately two cases, one where the eigenvalues are real and another where they are complex. In both cases we use the lemma below to obtain the estimates that we need. For the proof of the next result one may follow as in Gauer Palma (2013).

Lemma 2.1. Let $k>-n, \beta>0$ and $a>0$. Then

$$
\int_{|\xi| \leq \varepsilon} e^{-a|\xi|^{\beta} t}|\xi|^{k} d \xi \lesssim(1+t)^{-\frac{n+k}{\beta}}, \quad \forall t>0
$$

\subsection{Case $\alpha>2 \theta$ : real eigenvalues}

We consider $\theta \in\left[0, \frac{\alpha}{2}\right)$ and $\varepsilon>0$ defined by $\varepsilon^{\alpha-2 \theta}=\frac{1}{4}$. For $|\xi|<\varepsilon$ we have $|\xi|^{2(\alpha-2 \theta)}<\frac{1}{16}$ and thus the eigenvalues are real. To estimate $I_{0}$ and $I_{1}$ we use the equivalences on the eigenvalues given by the following lemma. Note that $\delta$ has no influence on the behavior of eigenvalues in the low frequency region (see also Lemma 2.4).

Lemma 2.2. If $|\xi|<\varepsilon$ then:

(i) $\lambda_{+} \approx-|\xi|^{2(\alpha-\theta)}$ (more precisely, $-4(2-\sqrt{2})|\xi|^{2(\alpha-\theta)} \leq \lambda_{+} \leq-|\xi|^{2(\alpha-\theta)}$;

(ii) $\lambda_{-} \approx-|\xi|^{2 \theta}$ (more precisely, $-|\xi|^{2 \theta} \leq \lambda_{-} \leq-\frac{1}{4}\left(1+\frac{1}{\sqrt{2}}\right)|\xi|^{2 \theta}$ );

(iii) $\lambda_{+}-\lambda_{-} \approx|\xi|^{2 \theta}$.

Proof:

(i) First, we note that 


$$
|\xi|^{2(\alpha-2 \theta)}<\frac{1}{16}=\frac{4(2-\sqrt{2})-2}{16(2-\sqrt{2})^{2}} .
$$

Multiplying the above estimate by $4|\xi|^{2(\alpha-2 \theta)}$ it implies that

$$
\begin{gathered}
-16(2-\sqrt{2})|\xi|^{2(\alpha-2 \theta)}+64(2-\sqrt{2})^{2}|\xi|^{4(\alpha-2 \theta)} \leq-8|\xi|^{2(\alpha-2 \theta)} \\
\leq-4|\xi|^{2(\alpha-2 \theta)}\left(1+|\xi|^{2 \delta}\right) .
\end{gathered}
$$

Using that $1-16(2-\sqrt{2})|\xi|^{2(\alpha-2 \theta)}+64(2-\sqrt{2})^{2}|\xi|^{4(\alpha-2 \theta)}=$ $\left(1-8(2-\sqrt{2})|\xi|^{2(\alpha-2 \theta)}\right)^{2}$ and $1-8(2-\sqrt{2})|\xi|^{2(\alpha-2 \theta)}>0$, we obtain

$$
-8(2-\sqrt{2})|\xi|^{2(\alpha-2 \theta)} \leq-1+\sqrt{1-4|\xi|^{2(\alpha-2 \theta)}\left(1+|\xi|^{2 \delta}\right)} .
$$

From $-\frac{1}{2} \leq-\frac{1}{2\left(1+|\xi|^{2 \delta}\right)}$ we conclude that

$$
-4(2-\sqrt{2})|\xi|^{2(\alpha-\theta)} \leq \frac{|\xi|^{2 \theta}}{2\left(1+|\xi|^{2 \delta}\right)}\left(-1+\sqrt{1-4|\xi|^{2(\alpha-2 \theta)}\left(1+|\xi|^{2 \delta}\right)}\right)=\lambda_{+} .
$$

To prove that $\lambda_{+} \leq-|\xi|^{2(\alpha-\theta)}$ we see that

$$
\left(-1+\sqrt{1-4|\xi|^{2(\alpha-2 \theta)}\left(1+|\xi|^{2 \delta}\right)}\right) \leq-2|\xi|^{2(\alpha-2 \theta)}\left(1+|\xi|^{2 \delta}\right)
$$

because $1-4|\xi|^{2(\alpha-2 \theta)}\left(1+|\xi|^{2 \delta}\right) \leq\left(1-2|\xi|^{2(\alpha-2 \theta)}\left(1+|\xi|^{2 \delta}\right)\right)^{2}$ and

$1-2|\xi|^{2(\alpha-2 \theta)}\left(1+|\xi|^{2 \delta}\right) \geq 0$. Multiplying both sides of above estimate by $\frac{|\xi|^{2 \theta}}{2\left(1+|\xi|^{2 \delta}\right)}$ we complete the proof.

(ii) From $\frac{1}{2} \leq 1-8|\xi|^{2(\alpha-2 \theta)} \leq 1-4|\xi|^{2(\alpha-2 \theta)}\left(1+|\xi|^{2 \delta}\right)$ we can claim that

$$
\frac{1}{4}\left(1+\frac{1}{\sqrt{2}}\right) \leq \frac{1}{2\left(1+|\xi|^{2 \delta}\right)}\left(1+\sqrt{1-4|\xi|^{2(\alpha-2 \theta)}\left(1+|\xi|^{2 \delta}\right)}\right) \leq 1 .
$$


To conclude the proof we multiply both sides by $-|\xi|^{2 \theta}$.

(iii) By the choice of $\varepsilon$, we have $|\xi|^{2(\alpha-2 \theta)}\left(1+|\xi|^{2 \delta}\right) \leq \frac{1}{8}$. Thus

$$
-\frac{1}{2} \leq-4|\xi|^{2(\alpha-2 \theta)}\left(1+|\xi|^{2 \delta}\right) \leq 0
$$

Therefore

$$
\frac{|\xi|^{2 \theta}}{2 \sqrt{2}} \leq \frac{|\xi|^{2 \theta}}{1+|\xi|^{2 \delta}} \sqrt{1-4|\xi|^{2(\alpha-2 \theta)}\left(1+|\xi|^{2 \delta}\right)} \leq|\xi|^{2 \theta}
$$

Lemma 2.3. Let $I_{0}$ and $I_{1}$ given by (9) and (10), $n \geq 1$ and $\gamma$ multi-index. Then for all $t \geq 0$ we have:

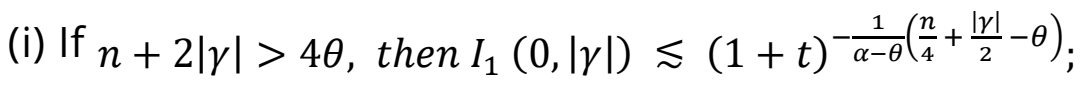

(ii) $I_{1}(1,|\gamma|) \lesssim\left\{\begin{array}{c}(1+t)^{-\frac{1}{\theta}\left(\frac{n}{4}+\frac{|\gamma|}{2}\right)}+(1+t)^{-\frac{1}{\alpha-\theta}\left(\frac{n}{4}+\frac{|\gamma|}{2}-\theta\right)-1} \\ (1+t)^{-\frac{1}{\alpha-\theta}\left(\frac{n}{4}+\frac{|\gamma|}{2}-\theta\right)-1}\end{array}\right.$ if $\theta>0$

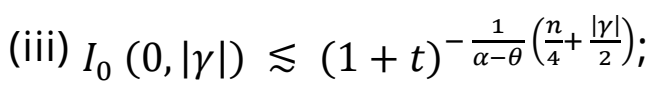
if $\theta=0$;

(iv) $I_{0}(1,|\gamma|) \lesssim(1+t)^{-\frac{1}{\alpha-\theta}\left(\frac{n}{4}+\frac{|\gamma|}{2}\right)-1}$.

Proof: We will estimate (10) to obtain (i) and (ii). The items (iii) and (iv) can be proved in the same way using (9).

(i) From Lemma 2.2 follow that

$$
\frac{\left|e^{\lambda_{+} t}-e^{\lambda_{-} t}\right|^{2}}{\left|\lambda_{+}-\lambda_{-}\right|^{2}} \lesssim \frac{e^{-2|\xi|^{2(\alpha-\theta)} t}}{|\xi|^{4 \theta}} .
$$

Using the above estimate and the Lemma 2.1 we obtain

$$
I_{1}^{2}(0,|\gamma|) \lesssim \int_{|\xi|<\varepsilon} e^{-2|\xi|^{2(\alpha-\theta)} t}|\xi|^{2|\gamma|-4 \theta} d \xi \lesssim(1+t)^{-\frac{n+2|\gamma|-4 \theta}{2(\alpha-\theta)}}
$$

because $n+2|\gamma|-4 \theta>0$.

(ii) Newly, using the Lemma 2.2,

$$
\frac{\left|\partial_{t}\left(e^{\lambda_{+} t_{-}} e^{\lambda_{-} t}\right)\right|^{2}}{\left|\lambda_{+}-\lambda_{-}\right|^{2}} \lesssim \frac{\lambda_{+}^{2} e^{2 \lambda_{+} t_{+}} \lambda_{-}^{2} e^{2 \lambda_{-} t}}{\left|\lambda_{+}-\lambda_{-}\right|^{2}} \lesssim|\xi|^{4(\alpha-2 \theta)} e^{-2|\xi|^{2(\alpha-\theta)} t}+e^{-c|\xi|^{2 \theta} t},
$$


with $c=\frac{1}{2}\left(1+\frac{1}{\sqrt{2}}\right)$.

In the same way it was done in (i), we get for $\theta \in\left(0, \frac{\alpha}{2}\right)$

$$
\begin{aligned}
I_{1}^{2}(1,|\gamma|) \lesssim & \int_{|\xi|<\varepsilon}\left(|\xi|^{4(\alpha-2 \theta)} e^{-2|\xi|^{2(\alpha-\theta)} t}+e^{-c|\xi|^{2 \theta} t}\right)|\xi|^{2|\gamma|} d \xi \\
& \lesssim(1+t)^{-\frac{n+2|\gamma|+4(\alpha-2 \theta)}{2(\alpha-\theta)}}+(1+t)^{-\frac{n+2|\gamma|}{2 \theta}} .
\end{aligned}
$$

The case $\theta=0$ is immediate.

Using the previous lemma we prove the following result to the real case for low frequency:

Proposition 2.1. Let $\alpha, \theta$ such that $0 \leq 2 \theta<\alpha, \gamma$ a multi-index and $\left(v_{0}, v_{1}\right) \in$ $L^{1}\left(\mathbb{R}^{n}\right) \times L^{1}\left(\mathbb{R}^{n}\right)$. Then the following estimates are true, for all $t \geq 0$ :

(i) If $n+2|\gamma|>4 \theta$ and $j=0,1$ then

$$
\begin{aligned}
\left\|\partial_{t}^{j} \partial_{x}^{\gamma} E_{0}(t)\right\| & \lesssim\left\|v_{0}\right\|_{L^{1}}(1+t)^{-\frac{1}{\alpha-\theta}\left(\frac{n}{4}+\frac{|\gamma|}{2}\right)-j} \\
& +\left\|v_{1}\right\|_{L^{1}}(1+t)^{-\frac{1}{\alpha-\theta}\left(\frac{n}{4}+\frac{|\gamma|}{2}-\theta\right)-j}
\end{aligned}
$$

(ii) If $n+2|\gamma| \leq 4 \theta$ then

$$
\left\|\partial_{t} \partial_{x}^{\gamma} E_{0}(t)\right\| \lesssim\left\|v_{0}\right\|_{L^{1}}(1+t)^{-\frac{1}{\alpha-\theta}\left(\frac{n}{4}+\frac{|\gamma|}{2}\right)-1}+\left\|v_{1}\right\|_{L^{1}}(1+t)^{-\frac{1}{\theta}\left(\frac{n}{4}+\frac{|\gamma|}{2}\right)} .
$$

Proof: For $j=0$ the item (i) follows directly from items (i) and (iii) of Lemma 2.3 and from estimate (11). To prove the item (ii), we choose $j=1$ in (11) and we use the Lemma 2.3, items (ii) and (iv). To obtain the best decay rate given in (ii), we see that $n+2|\gamma| \leq 4 \theta$ implies

$$
\frac{n+2|\gamma|+4(\alpha-2 \theta)}{2(\alpha-\theta)} \geq \frac{n+2|\gamma|}{2 \theta} .
$$

Thus (13) is true. On the other hand, if $n+2|\gamma|>4 \theta$ we have the opposite inequality. This implies the estimate (12) with $j=1$. 


\subsection{Case $\alpha \leq 2 \theta$ complex eigenvalues}

Now we consider $\theta \epsilon\left[\frac{\alpha}{2}, \alpha\right]$ and $\varepsilon \epsilon(0,1)$. In this case we have complex eigenvalues, given by

$$
\lambda_{ \pm}=\frac{|\xi|^{2 \theta}}{2\left(1+|\xi|^{2 \delta}\right)}\left(-1 \pm i \sqrt{4|\xi|^{2(\alpha-2 \theta)}\left(1+|\xi|^{2 \delta}\right)-1}\right)
$$

We obtain the results for complex case following the same steps of the real case.

Lemma 2.4. If $|\xi|<\varepsilon$ then:

(i) $\left|\lambda_{+}-\lambda_{-}\right| \approx|\xi|^{\alpha}$;

(ii) $\left|\lambda_{ \pm}\right|^{2} \lesssim|\xi|^{2 \alpha}$;

(iii) $\left|e^{\lambda_{ \pm} t}\right| \lesssim e^{-\frac{1}{4}|\xi|^{2 \theta} t}$.

Proof:

(i) Using $1 \leq 1+|\xi|^{2 \delta} \leq 2$, the proof follows directly from the inequality $\frac{1}{8}\left(4|\xi|^{2(\alpha-2 \theta)}\left(1+|\xi|^{2 \delta}\right)-1\right) \leq|\xi|^{2(\alpha-2 \theta)} \leq 4|\xi|^{2(\alpha-2 \theta)}\left(1+|\xi|^{2 \delta}\right)-1, \forall 0<|\xi|<\varepsilon$.

(iii) By Re $\left(\lambda_{ \pm}\right) \leq-\frac{1}{4}|\xi|^{2 \theta}$ we have $\left|e^{\lambda_{ \pm} t}\right| \leq e^{R e\left(\lambda_{ \pm}\right) t} \leq e^{-\frac{1}{4}|\xi|^{2 \theta} t}$.

The proof of the next lemma is quite similar to that of Lemma 2.3. We omit the details.

Lemma 2.5. Let $I_{0}$ and $I_{1}$ given by (9) and (10), $n \geq 1$ and $\gamma$ multi-index. Then, for all $t \geq 0$ we have for $\theta>0$ :

(i) If $n+2|\gamma|>2 \alpha$, then $I_{1}(0,|\gamma|) \lesssim(1+t)^{-\frac{1}{\theta}\left(\frac{n}{4}+\frac{|\gamma|}{2}-\frac{\alpha}{2}\right)}$;

(ii) $I_{1}(1,|\gamma|) \lesssim(1+t)^{-\frac{1}{\theta}\left(\frac{n}{4}+\frac{|\gamma|}{2}\right)}$;

(iii) $I_{0}(0,|\gamma|) \lesssim(1+t)^{-\frac{1}{\theta}\left(\frac{n}{4}+\frac{|\gamma|}{2}\right)}$;

(iv) $I_{0}(1,|\gamma|) \lesssim(1+t)^{-\frac{1}{\theta}\left(\frac{n}{4}+\frac{|\gamma|}{2}+\frac{\alpha}{2}\right)}$.

If $\alpha=\theta=0$ then it is immediate that $I_{i}(j,|\gamma|) \lesssim e^{-\frac{t}{4}}, t \geq 0$, for all $i, j \in\{0,1\}$.

From estimate (11) and Lemma 2.5, we have the following result: 
Proposition 2.2. Let $\delta, \alpha \geq 0, \theta \in\left[\frac{\alpha}{2}, \alpha\right], \gamma$ a multi-index and $\left(v_{0}, v_{1}\right) \in L^{1}\left(\mathbb{R}^{n}\right) \times$ $L^{1}\left(\mathbb{R}^{n}\right)$. Then, for $t \geq 0$, the following estimates are true:

(i) If $n+2|\gamma|>2 \alpha$ and $\theta>0$, then

$$
\left\|\partial_{x}^{\gamma} E_{0}(t)\right\| \lesssim\left\|v_{0}\right\|_{L^{1}}(1+t)^{-\frac{1}{\theta}\left(\frac{n}{4}+\frac{|\gamma|}{2}\right)}+\left\|v_{1}\right\|_{L^{1}}(1+t)^{-\frac{1}{\theta}\left(\frac{n}{4}+\frac{|\gamma|}{2}-\frac{\alpha}{2}\right)}
$$

(ii) If $n \geq 1$ and $\theta>0$, then

$$
\left\|\partial_{t} \partial_{x}^{\gamma} E_{0}(t)\right\| \lesssim\left\|v_{0}\right\|_{L^{1}}(1+t)^{-\frac{1}{\theta}\left(\frac{n}{4}+\frac{|\gamma|}{2}+\frac{\alpha}{2}\right)}+\left\|v_{1}\right\|_{L^{1}}(1+t)^{-\frac{1}{\theta}\left(\frac{n}{4}+\frac{|\gamma|}{2}\right)}
$$

(iii) If $\alpha=\theta=0$ then $\left\|\partial_{t}^{j} \partial_{x}^{\gamma} E_{0}(t)\right\| \lesssim\left\{\left\|v_{0}\right\|_{L^{1}}+\left\|v_{1}\right\|_{L^{1}}\right\} e^{-\frac{t}{4}}$, for $j=0,1$.

\section{HIGH FREQUENCY REGION: $|\xi| \geq \varepsilon$}

The results obtained in the previous section complete the estimates we need to prove the Theorems 4.1 and 4.2 in the region of low frequency. Next, we are going to obtain estimates for the region of high frequency and the required regularity at initial data to obtain the desired decay. For this purpose, we apply the multiplier method in Fourier space. Note that the technique used in this section is suitable for $L^{2}-L^{2}$ estimates. To obtain estimates of the type $L^{p}-L^{q}$ another technique would need to be used.

We consider, for $|\xi| \geq \varepsilon$, (with $\varepsilon \epsilon(0,1)$ defined at previous section), the following auxiliary function:

$$
\rho(\xi)= \begin{cases}\frac{\varepsilon^{2 \alpha+2 \delta-4 \theta}|\xi|^{2 \theta}}{2\left(1+|\xi|^{2 \delta}\right)}, & \text { if } \alpha+\delta \geq 2 \theta \\ \frac{\varepsilon^{-2 \alpha+4 \theta}|\xi|^{2 \alpha-2 \theta}}{4}, & \text { if } \alpha+\delta<2 \theta .\end{cases}
$$

It is easy to prove that

$$
\rho(\xi) \leq \frac{|\xi|^{2 \theta}}{2\left(1+|\xi|^{2 \delta}\right)} \text { and } \rho(\xi) \leq \frac{|\xi|^{2 \alpha-2 \theta}}{2}
$$


for $|\xi| \geq \varepsilon$

We denote by $E_{1}(t)$ the energy of order $\sigma$ of the equation (4) in the Fourier space given by

$$
E_{1}(t)=\frac{1}{2}|\xi|^{2 \delta+\sigma}\left|\hat{v}_{t}(t)\right|^{2}+\frac{1}{2}|\xi|^{2 \alpha+\sigma}|\hat{v}(t)|^{2}, \quad t \geq 0 .
$$

Therefore

$$
2 \int_{|\xi| \geq \varepsilon} E_{1}(t) d \xi=\int_{\mathbb{R}^{n}}(1-\chi(\xi))^{2}\left\{|\xi|^{2 \delta+\sigma}\left|\hat{v}_{t}(t)\right|^{2}+|\xi|^{2 \alpha+\sigma}|\hat{v}(t)|^{2}\right\} d \xi
$$

where $\chi(\xi)$ is the characteristic function of $\left\{\xi \in \mathbb{R}^{n} /|\xi|<\varepsilon\right\}$.

Note that choosing $\sigma$ in a suitable way and using Plancherel theorem, the above integral becames the $L^{2}$-norm of derivatives of $E_{\infty}$ and $\partial_{t} E_{\infty}$ (see definition of $E_{\infty}$ at (7)). Thus our goal in this section is to estimate $\int_{|\xi| \geq \varepsilon} E_{1}(t) d \xi$.

Next we show and prove the main result of this section. Note that the decay estimate (ii) in the next proposition is due to the regularity-loss structure of the equation for $\theta<\delta$. In fact, to obtain decay rates to the energy in high frequency region we need to impose more regularity on the initial data. The decay rate is directly related to the additional regularity in the initial data $\left(\frac{\delta-\theta}{\beta}\right.$, with $\left.\theta<\delta\right)$. Thus, to improve the decay rate it is necessary to take smaller $\beta$, but this imply that it is necessary additional regularity on the initial data. This does not occur in item (i).

Proposition 3.1. Let $\delta, \alpha \geq 0, \theta \in[0, \alpha]$ and $\sigma \in \mathbb{R}$.

(i) If $\left(v_{0}, v_{1}\right) \in H^{\alpha+\frac{\sigma}{2}}\left(\mathbb{R}^{n}\right) \times H^{\delta+\frac{\sigma}{2}}\left(\mathbb{R}^{n}\right)$ and $\delta \leq \theta \leq \alpha$, then there is a constant $c>0$ such that

$$
\int_{|\xi| \geq \varepsilon} E_{1}(t, \xi) d \xi \lesssim\left\{\left\|v_{1}\right\|_{H^{\delta+\frac{\sigma}{2}}}^{2}+\left\|v_{0}\right\|_{H^{\alpha+\frac{\sigma}{2}}}^{2}\right\} e^{-c t}, \quad \forall t \geq 0
$$

(ii) If $\left(v_{0}, v_{1}\right) \in H^{s}\left(\mathbb{R}^{n}\right) \times H^{r}\left(\mathbb{R}^{n}\right), \beta>0$ and $\theta<\delta$ then there is $C=C(\beta)>0$ such that 


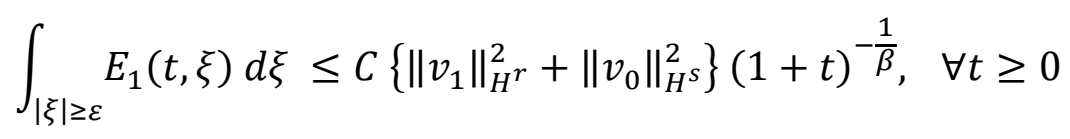

with $s=\alpha+\frac{\delta-\theta}{\beta}+\frac{\sigma}{2}$ and $r=\delta+\frac{\delta-\theta}{\beta}+\frac{\sigma}{2}$.

To prove Proposition 3.1 we need several estimates and lemmas on the solution $\hat{v}(t, \xi)$ of the corresponding problem (4) in the Fourier space. Multiplying equation (4) by $|\xi|^{\sigma} \overline{\hat{v}}_{t}+\rho(\xi)|\xi|^{\sigma} \overline{\hat{v}}$ and taking the real part we have

$$
\frac{d}{d t} E(t)+F(t)=R(t), \quad t \geq 0
$$

where

$$
\begin{aligned}
E(t, \xi)= & \frac{1}{2}\left(1+|\xi|^{2 \delta}\right)|\xi|^{\sigma}\left|\hat{v}_{t}(t)\right|^{2}+\frac{1}{2}|\xi|^{2 \alpha+\sigma}|\hat{v}(t)|^{2}+\rho(\xi)\left(1+|\xi|^{2 \delta}\right)|\xi|^{\sigma} \operatorname{Re}\left\{\hat{v}_{t}(t) \hat{v}(t)\right\} \\
& +\frac{1}{2} \rho(\xi)|\xi|^{2 \theta+\sigma}|\hat{v}(t)|^{2} \\
F(t, \xi)= & |\xi|^{2 \theta+\sigma}\left|\hat{v}_{t}(t)\right|^{2}+\rho(\xi)|\xi|^{2 \alpha+\sigma}|\hat{v}(t)|^{2} \\
R(t, \xi)= & \rho(\xi)\left(1+|\xi|^{2 \delta}\right)|\xi|^{\sigma}\left|\hat{v}_{t}(t)\right|^{2}
\end{aligned}
$$

By (15) it follows that $R(t) \leq \frac{1}{2} F(t)$. Substituting this estimate into (16), we get

$$
\frac{d}{d t} E(t)+\frac{1}{2} F(t) \leq 0
$$

for all $t \geq 0$ and $|\xi| \geq \varepsilon$.

Lemma 3.1. The functionals $E(t, \xi)$ and $E_{1}(t, \xi)$ are equivalent for all $t \geq 0$ and $|\xi| \geq \varepsilon$.

Proof: First, we note that

$$
\begin{gathered}
\pm \rho(\xi)\left(1+|\xi|^{2 \delta}\right) \operatorname{Re}\left\{\hat{v}_{t}(t) \overline{\hat{v}}(t)\right\} \leq\left(1+|\xi|^{2 \delta}\right) \frac{\left|\hat{v}_{t}(t)\right|^{2}}{2 \eta}+\rho(\xi)^{2}\left(1+|\xi|^{2 \delta}\right) \frac{\eta|\hat{v}(t)|^{2}}{2} \leq \\
\left(1+|\xi|^{2 \delta}\right) \frac{\left|\hat{v}_{t}(t)\right|^{2}}{2 \eta}+|\xi|^{2 \alpha} \frac{\eta|\hat{v}(t)|^{2}}{8}
\end{gathered}
$$


because, by (15),

$$
\rho(\xi)^{2}\left(1+|\xi|^{2 \delta}\right) \leq \frac{|\xi|^{2 \theta}}{2\left(1+|\xi|^{2 \delta}\right)} \frac{|\xi|^{2 \alpha-2 \theta}}{2}\left(1+|\xi|^{2 \delta}\right)=\frac{|\xi|^{2 \alpha}}{4}
$$

Using (18) with positive sign and $\eta=1$, we have:

$$
E(t) \lesssim|\xi|^{\sigma}\left(\frac{1}{2}|\xi|^{2 \delta}\left|\hat{v}_{t}(t)\right|^{2}+\frac{1}{2}|\xi|^{2 \alpha}|\hat{v}(t)|^{2}\right)=E_{1}(t)
$$

since, by (15), $\rho(\xi)|\xi|^{2 \theta} \leq \frac{1}{2}|\xi|^{2 \alpha}$ and $1+|\xi|^{2 \delta} \leq\left(\varepsilon^{-2 \delta}+1\right)|\xi|^{2 \delta}$.

On the other hand, using (18) with negative sign and $\eta=2$, we have:

$$
E(t) \geq|\xi|^{\sigma}\left(\frac{1}{4}|\xi|^{2 \delta}\left|\hat{v}_{t}(t)\right|^{2}+\frac{1}{4}|\xi|^{2 \alpha}|\hat{v}(t)|^{2}\right)=\frac{1}{2} E_{1}(t)
$$

Lemma 3.2. If $\delta \leq \theta \leq \alpha$ then $E_{1}(t, \xi) \lesssim F(t, \xi)$ for all $t \geq 0$ and $|\xi| \geq \varepsilon$.

Proof: For all $t \geq 0$ and $|\xi| \geq \varepsilon$, we have

$$
|\xi|^{2 \delta}\left|\hat{v}_{t}(t)\right|^{2} \lesssim|\xi|^{2 \theta}\left|\hat{v}_{t}(t)\right|^{2}
$$

and

$$
|\xi|^{2 \alpha}|\hat{v}(t)|^{2} \lesssim \rho(\xi)|\xi|^{2 \alpha}|\hat{v}(t)|^{2}
$$

The last inequality actually works, because the case $\alpha+\delta \geq 2 \theta$ follows from (14) using the hypothesis $\delta \leq \theta$; and the case $\alpha+\delta<2 \theta$ follows from $|\xi|^{2 \alpha} \gtrsim|\xi|^{2 \theta}$, which works by hypothesis $\theta \leq \alpha$.

Multiplying (19) and (20) by $|\xi|^{\sigma}$ and adding the two resulting inequalities, the lemma is proved.

Using the estimate (17) and the Lemmas 3.1 and 3.2, we can prove that there are positive constants $c, c_{1}$ such that

$$
\frac{d}{d t} E(t)+c E(t) \leq \frac{d}{d t} E(t)+c_{1} E_{1}(t) \leq \frac{d}{d t} E(t)+\frac{1}{2} F(t) \leq 0, \quad \forall t \geq 0 \text { and }|\xi| \geq \varepsilon
$$


By the equivalence between $E$ and $E_{1}$ given by Lemma 3.1 we conclude that

$$
E_{1}(t, \xi) \lesssim e^{-c t} E_{1}(0, \xi), \quad \forall t \geq 0 \text { and }|\xi| \geq \varepsilon .
$$

Integrating over the region of high frequency $|\xi| \geq \varepsilon$, we obtain

$$
\begin{aligned}
\int_{|\xi| \geq \varepsilon} E_{1}(t, \xi) d \xi & \lesssim e^{-c t} \int_{|\xi| \geq \varepsilon}\left\{|\xi|^{2 \delta+\sigma}\left|\hat{v}_{1}\right|^{2}+|\xi|^{2 \alpha+\sigma}\left|\hat{v}_{0}\right|^{2}\right\} d \xi \\
& \lesssim e^{-c t} \int_{|\xi| \geq \varepsilon}\left(1+|\xi|^{2}\right)^{\delta+\frac{\sigma}{2}}\left|\hat{v}_{1}\right|^{2} d \xi \\
& +\int_{|\xi| \geq \varepsilon}\left(1+|\xi|^{2}\right)^{\alpha+\frac{\sigma}{2}}\left|\hat{v}_{0}\right|^{2} d \xi \lesssim\left\{\left\|v_{1}\right\|_{H^{\delta+\frac{\sigma}{2}}}^{2}+\left\|v_{0}\right\|_{H^{\alpha+\frac{\sigma}{2}}}^{2}\right\} e^{-c t}
\end{aligned}
$$

The proof of the item ( $i$ ) of Proposition 3.1 is complete.

The next lemma is important to estimate the integral of energy in high frequency region in the Fourier space when there is the property of regularityloss, that is, when $\theta<\delta$. Using the result of next lemma we will prove the item (ii) of Proposition 3.1.

Lemma 3.3. We define

$$
I(t)=\int_{|\xi| \geq \varepsilon} E_{1}(t, \xi) d \xi \quad \text { and } \quad J(t)=\int_{|\xi| \geq \varepsilon} F(t, \xi) d \xi \text {. }
$$

Let $\delta, \alpha \geq 0, \theta \in[0, \alpha], \sigma \in \mathbb{R}, \beta>0, \theta<\delta$ and $\left(v_{0}, v_{1}\right) \in H^{s}\left(\mathbb{R}^{n}\right) \times H^{r}\left(\mathbb{R}^{n}\right)$. Then there is $C_{\beta}>0$ such that, for all $t \geq 0$,

$$
[I(t)]^{1+\beta} \leq C_{\beta}\left\{\left\|v_{1}\right\|_{H^{r}}+\left\|v_{0}\right\|_{H^{s}}\right\}^{\beta} J(t)
$$

with $s=\alpha+\frac{\delta-\theta}{\beta}+\frac{\sigma}{2}$ and $r=\delta+\frac{\delta-\theta}{\beta}+\frac{\sigma}{2}$.

Proof: For any $\beta>0$ we have 


$$
\begin{aligned}
& {[I(t)]^{1+\beta} \lesssim\left[\int_{|\xi| \geq \varepsilon}|\xi|^{2 \delta+\sigma}\left|\hat{v}_{t}(t)\right|^{2} d \xi\right]^{1+\beta}+\left[\int_{|\xi| \geq \varepsilon}|\xi|^{2 \alpha+\sigma}|\hat{v}(t)|^{2} d \xi\right]^{1+\beta}} \\
& =\left[\int_{|\xi| \geq \varepsilon}|\xi|^{-\frac{2 \theta+\sigma}{1+\beta}}|\xi|^{2 \delta+\sigma}\left|\hat{v}_{t}(t)\right|^{2-\frac{2}{1+\beta}}|\xi|^{\frac{2 \theta+\sigma}{1+\beta}}\left|\hat{v}_{t}(t)\right|^{\frac{2}{1+\beta}} d \xi\right]^{1+\beta} \\
& +\left[\int_{|\xi| \geq \varepsilon}\left(\rho(\xi)|\xi|^{2 \alpha+\sigma}\right)^{-\frac{1}{1+\beta}}|\xi|^{2 \alpha+\sigma}|\hat{v}(t)|^{2-\frac{2}{1+\beta}}\left(\rho(\xi)|\xi|^{2 \alpha+\sigma}\right)^{\frac{1}{1+\beta}}|\hat{v}(t)|^{\frac{2}{1+\beta}} d \xi\right]^{1+\beta}
\end{aligned}
$$

for all $t \geq 0$. Then, by using Hölder's inequality in $L^{\frac{1+\beta}{\beta}}$ and $L^{1+\beta}$ we obtain

$[I(t)]^{1+\beta} \lesssim\left\{\left[\int_{|\xi| \geq \varepsilon}|\xi|^{-\frac{2 \theta}{\beta}+\sigma+\frac{2 \delta}{\beta}+2 \delta}\left|\hat{v}_{t}(t)\right|^{2} d \xi\right]^{\beta}+\left[\int_{|\xi| \geq \varepsilon} \rho(\xi)^{-\frac{1}{\beta}}|\xi|^{2 \alpha+\sigma}|\hat{v}(t)|^{2} d \xi\right]^{\beta}\right\} J(t)$

for all $t \geq 0$.

Next we need to estimate both integrals in the right hand side of the last inequality in terms of the initial data. Multiplying equation (4) by $\overline{\hat{v}}_{t}$, taking the real part on the resulting identity and integrating over the time interval $(0, t)$ we obtain

$$
\begin{aligned}
& \left(1+|\xi|^{2 \delta}\right)\left|\hat{v}_{t}(t)\right|^{2}+|\xi|^{2 \alpha}|\hat{v}(t)|^{2} \\
& +2 \int_{0}^{t}|\xi|^{2 \theta}\left|\hat{v}_{t}(s)\right|^{2} d s=\left(1+|\xi|^{2 \delta}\right)\left|\hat{v}_{1}\right|^{2}+|\xi|^{2 \alpha}\left|\hat{v}_{0}\right|^{2}
\end{aligned}
$$

Now, multiplying both sides of the above identity by $|\xi|^{-\frac{2 \theta}{\beta}+\sigma+\frac{2 \delta}{\beta}}$ and integrating over $|\xi| \geq \varepsilon$ we obtain

$$
\begin{array}{r}
\int_{|\xi| \geq \varepsilon}|\xi|^{-\frac{2 \theta}{\beta}+\sigma+\frac{2 \delta}{\beta}+2 \delta}\left|\hat{v}_{t}(t)\right|^{2} d \xi+\int_{|\xi| \geq \varepsilon}|\xi|^{-\frac{2 \theta}{\beta}+\sigma+\frac{2 \delta}{\beta}+2 \alpha}|\hat{v}(t)|^{2} d \xi \lesssim \\
\int_{|\xi| \geq \varepsilon}\left(1+|\xi|^{2}\right)^{\delta+\frac{\delta-\theta}{\beta}+\frac{\sigma}{2}}\left|\hat{v}_{1}\right|^{2} d \xi+\int_{|\xi| \geq \varepsilon}\left(1+|\xi|^{2}\right)^{\alpha+\frac{\delta-\theta}{\beta}+\frac{\sigma}{2}}\left|\hat{v}_{0}\right|^{2} d \xi .
\end{array}
$$

By the assumptions of lemma we have $\alpha+\delta \geq 2 \theta$. Then, by definition of $\rho(\xi)$ given in (14) it follows that 


$$
\int_{|\xi| \geq \varepsilon} \rho(\xi)^{-\frac{1}{\beta}}|\xi|^{2 \alpha+\sigma}|\hat{v}(t)|^{2} d \xi \leq K_{\beta} \int_{|\xi| \geq \varepsilon}|\xi|^{-\frac{2 \theta}{\beta}+\sigma+\frac{2 \delta}{\beta}+2 \alpha}|\hat{v}(t)|^{2} d \xi
$$

Using (22)-(23) in (21), we obtain

$$
[I(t)]^{1+\beta} \leq C_{\beta}\left\{\left\|v_{1}\right\|_{H^{r}}^{2}+\left\|v_{0}\right\|_{H^{s}}^{2}\right\}^{\beta} J(t), \quad \forall t \geq 0,
$$

with $r=\delta+\frac{\delta-\theta}{\beta}+\frac{\sigma}{2}$ and $s=\alpha+\frac{\delta-\theta}{\beta}+\frac{\sigma}{2}$.

Now, we observe that, by Lemma 3.3, we can write

$$
\frac{K}{2}[I(t)]^{1+\beta} \leq \frac{1}{2} J(t), \quad \forall t \geq 0,
$$

where $K=\left(C_{\beta}\left\{\left\|v_{1}\right\|_{H^{r}}^{2}+\left\|v_{0}\right\|_{H^{s}}^{2}\right\}^{\beta}\right)^{-1}$.

By Lemma 3.1, there are constants $m$ and $M$ such that

$$
m E_{1}(t, \xi) \leq E(t, \xi) \leq M E_{1}(t, \xi), \quad \forall t \geq 0 \text { and }|\xi| \geq \varepsilon .
$$

Consequently, we have

$$
\frac{d}{d t} \int_{|\xi| \geq \varepsilon} E(t) d \xi+\frac{K}{2 M^{1+\beta}}\left[\int_{|\xi| \geq \varepsilon} E(t) d \xi\right]^{1+\beta} \leq \frac{d}{d t} \int_{|\xi| \geq \varepsilon} E(t) d \xi+\frac{1}{2} J(t) \leq 0
$$

where the last inequality is due to the estimate (17).

Therefore, applying the Lyapunov's theorem (see Komornik (1996)) in (24), we conclude the following decay estimate

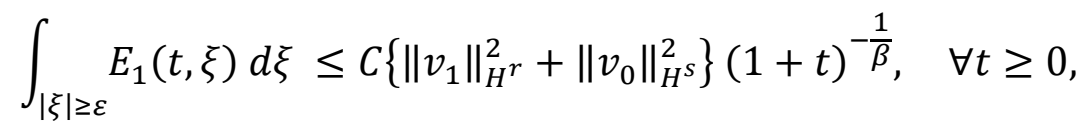

with $0<C=C(\beta)$. The proof of the item (ii) of Proposition 3.1 is complete.

\section{RESULTS AND APPLICATIONS}




\subsection{Main results}

In this section we use the results obtained in the previous sections in order to find decay rates for the $L^{2}$-norm of $\partial_{x}^{\gamma_{1}} v(t)$ and $\partial_{x}^{\gamma_{2}} v_{t}(t)$ and the corresponding regularity in the initial data. Observe that for appropriate choices of $\gamma_{1}$ and $\gamma_{2}$ we can obtain estimates for the $L^{2}$-norm of solution, for each term of energy defined in (3) and also for $H^{m}$ norms of $v$ and $v_{t}$. In Theorem 4.1 we do not determine values for $\beta>0$ for the purpose of to make this choice appropriately on each application. The same occurs in Theorem 4.2. We can choose $\beta$ so that give the sharp rates and in this case will require more of the regularity of the initial data. Or we can also choose $\beta$ to require less regularity on the initial data, resulting in worse rates.

Theorem 4.1. If $\delta, \alpha \geq 0, \theta \in\left[0, \frac{\alpha}{2}\right), v_{0} \in H^{s}\left(\mathbb{R}^{n}\right) \cap L^{1}\left(\mathbb{R}^{n}\right)$ and $v_{1} \in H^{r}\left(\mathbb{R}^{n}\right) \cap$ $L^{1}\left(\mathbb{R}^{n}\right)$, for $r$ and $s$ specified below, then the solution of the problem (1) - (2) satisfies the following estimates for all $t \geq 0$ :

(i) If $n+2\left|\gamma_{1}\right|>4 \theta$ then

$$
\begin{gathered}
\left\|\partial_{x}^{\gamma_{1}} v(t)\right\| \lesssim \\
+\left\{v_{0}\left\|_{L^{1}}(1+t)^{-\frac{1}{\alpha-\theta}\left(\frac{n}{4}+\frac{\left|\gamma_{1}\right|}{2}\right)}+\right\| v_{1} \|_{L^{1}}(1+t)^{-\frac{1}{\alpha-\theta}\left(\frac{n}{4}+\frac{\left|\gamma_{1}\right|}{2}-\theta\right)}\right. \\
+\left\{\left\|v_{1}\right\|_{H^{r}}+\left\|v_{0}\right\|_{H^{s}}\right\} f(t)
\end{gathered}
$$

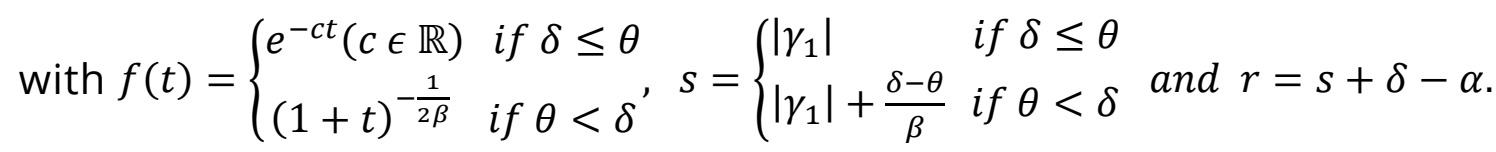

(ii) If $n+2\left|\gamma_{2}\right|<2 \alpha$ and $\theta \in\left[\frac{n}{4}+\frac{\left|\gamma_{2}\right|}{2}, \frac{\alpha}{2}\right)$ then

$$
\begin{aligned}
\left\|\partial_{x}^{\gamma_{2}} v_{t}(t)\right\| & \lesssim\left\|v_{0}\right\|_{L^{1}}(1+t)^{-\frac{1}{\alpha-\theta}\left(\frac{n}{4}+\frac{\left|\gamma_{2}\right|}{2}\right)-1}+\left\|v_{1}\right\|_{L^{1}}(1+t)^{-\frac{1}{\theta}\left(\frac{n}{4}+\frac{\left|\gamma_{2}\right|}{2}\right)} \\
& +\left\{\left\|v_{1}\right\|_{H^{r}}+\left\|v_{0}\right\|_{H^{s}}\right\} f(t),
\end{aligned}
$$

and if $n+2\left|\gamma_{2}\right| \geq 2 \alpha$ or $\theta \in\left[0, \frac{n}{4}+\frac{\left|\gamma_{2}\right|}{2}\right)$ then

$$
\left\|\partial_{x}^{\gamma_{2}} v_{t}(t)\right\| \lesssim\left\|v_{0}\right\|_{L^{1}}(1+t)^{-\frac{1}{\alpha-\theta}\left(\frac{n}{4}+\frac{\left|\gamma_{2}\right|}{2}\right)-1}+\left\|v_{1}\right\|_{L^{1}}(1+t)^{-\frac{1}{\alpha-\theta}\left(\frac{n}{4}+\frac{\left|\gamma_{2}\right|}{2}-\theta\right)-1}
$$




$$
+\left\{\left\|v_{1}\right\|_{H^{r}}+\left\|v_{0}\right\|_{H^{s}}\right\} f(t)
$$

with $f(t)=\left\{\begin{array}{ll}e^{-c t}(c \in \mathbb{R}) & \text { if } \delta \leq \theta \\ (1+t)^{-\frac{1}{2 \beta}} & \text { if } \theta<\delta\end{array}, \quad r=\left\{\begin{array}{ll}\left|\gamma_{2}\right| & \text { if } \delta \leq \theta \\ \left|\gamma_{2}\right|+\frac{\delta-\theta}{\beta} & \text { if } \theta<\delta\end{array}\right.\right.$ and $s=r+\alpha-\delta$.

Proof:

(i) By definition of $E_{0}$ and $E_{\infty}$ we have

$$
\left\|\partial_{x}^{\gamma_{1}} v(t)\right\| \leq\left\|\partial_{x}^{\gamma_{1}} E_{0}(t)\right\|+\left\|\partial_{x}^{\gamma_{1}} E_{\infty}(t)\right\| .
$$

We choose $\gamma=\gamma_{1}$ at Proposition 2.1 and consider $n+2\left|\gamma_{1}\right|>4 \theta$.Using (12) and the Proposition 3.1 with $\sigma=2\left|\gamma_{1}\right|-2 \alpha$ it follows the result.

(ii) We can write

$$
\left\|\partial_{x}^{\gamma_{2}} v_{t}(t)\right\| \leq\left\|\partial_{x}^{\gamma_{2}} \partial_{t} E_{0}(t)\right\|+\left\|\partial_{t} \partial_{x}^{\gamma_{2}} E_{\infty}(t)\right\| .
$$

Choosing $\gamma=\gamma_{2}$ at Proposition 2.1 and $\sigma=2\left|\gamma_{2}\right|-2 \delta$ at Proposition 3.1, we have two options. If $n+2\left|\gamma_{2}\right|<2 \alpha$ and $\theta \in\left[\frac{n}{4}+\frac{\left|\gamma_{2}\right|}{2}, \frac{\alpha}{2}\right)$ we use (13) and obtain (25). For the case $n+2\left|\gamma_{2}\right| \geq 2 \alpha$ or $\theta \in\left[0, \frac{n}{4}+\frac{\left|\gamma_{2}\right|}{2}\right)$ using (13) it follows (26).

In the same way, we prove the following result, for the case $\alpha \leq 2 \theta$ :

Theorem 4.2. If $\delta, \alpha \geq 0, \theta \in\left[\frac{\alpha}{2}, \alpha\right], v_{0} \in H^{s}\left(\mathbb{R}^{n}\right) \cap L^{1}\left(\mathbb{R}^{n}\right)$ and $v_{1} \in H^{r}\left(\mathbb{R}^{n}\right) \cap$ $L^{1}\left(\mathbb{R}^{n}\right)$, with $f(t), r$ and $s$ defined at same manner as in Theorem 4.1, the following estimates are true, for all $t \geq 0$ :

(i) If $n+2\left|\gamma_{1}\right|>2 \alpha$ then for $\theta>0$ we have

$$
\begin{gathered}
\left\|\partial_{x}^{\gamma_{1}} v(t)\right\| \lesssim \\
+\left\{v_{0}\left\|_{L^{1}}(1+t)^{-\frac{1}{\theta}\left(\frac{n}{4}+\frac{\left|\gamma_{1}\right|}{2}\right)}+\right\| v_{1}\left\|_{H^{r}}\right\|_{L^{1}}(1+t)^{-\frac{1}{\theta}\left(\frac{n}{4}+\frac{\left|\gamma_{1}\right|}{2}-\frac{\alpha}{2}\right)}\right. \\
\left.+\|_{H^{s}}\right\} f(t)
\end{gathered}
$$

and, for $\theta=0$,

$$
\left\|\partial_{x}^{\gamma_{1}} v(t)\right\| \lesssim\left\{\left\|v_{0}\right\|_{L^{1}}+\left\|v_{1}\right\|_{L^{1}}\right\} e^{-\frac{t}{4}}+\left\{\left\|v_{1}\right\|_{H^{r}}+\left\|v_{0}\right\|_{H^{s}}\right\} f(t)
$$


(ii) If $n \geq 1$ and $\theta>0$ then

$$
\begin{aligned}
\left\|\partial_{x}^{\gamma_{2}} v_{t}(t)\right\| \lesssim & \left\|v_{0}\right\|_{L^{1}}(1+t)^{-\frac{1}{\theta}\left(\frac{n}{4}+\frac{\left|\gamma_{2}\right|}{2}+\frac{\alpha}{2}\right)}+\left\|v_{1}\right\|_{L^{1}}(1+t)^{-\frac{1}{\theta}\left(\frac{n}{4}+\frac{\left|\gamma_{2}\right|}{2}\right)} \\
& +\left\{\left\|v_{1}\right\|_{H^{r}}+\left\|v_{0}\right\|_{H^{s}}\right\} f(t)
\end{aligned}
$$

and, if $\theta=0$,

$$
\left\|\partial_{x}^{\gamma_{2}} v_{t}(t)\right\| \lesssim\left\{\left\|v_{0}\right\|_{L^{1}}+\left\|v_{1}\right\|_{L^{1}}\right\} e^{-\frac{t}{4}}+\left\{\left\|v_{1}\right\|_{H^{r}}+\left\|v_{0}\right\|_{H^{s}}\right\} f(t) .
$$

\subsection{Applications}

Now let us apply the above results to several initial value problems associated with some dissipative partial differential equations of second order in time.

\subsubsection{Wave equation with fractional damping}

We consider the equation (1)-(2) with $\delta=0$ and $\alpha=1$, that is, the wave equation

$$
v_{t t}(t, x)-\Delta v(t, x)+(-\Delta)^{\theta} v_{t}(t, x)=0, \quad t \geq 0, \quad x \in \mathbb{R}^{n}
$$

with initial data

$$
v(0, x)=v_{0}(x), \quad v_{t}(0, x)=v_{1}(x)
$$

and $\theta \epsilon[0,1]$, where the associated energy is defined by

$$
E_{v}(t)=\frac{1}{2}\left\{\left\|v_{t}(t)\right\|^{2}+\|\nabla v(t)\|^{2}\right\}
$$


Note that $\delta \leq \theta$. Moreover, for this choice of $\alpha$, we have $\alpha>2 \theta$ if and only if $\theta<\frac{1}{2}$. Thus, it appears here the separation in cases $\theta \in\left[0, \frac{1}{2}\right.$ ) (real eigenvalues for the low frequency region) and $\theta \in\left[\frac{1}{2}, 1\right]$ (complex eigenvalues for the low frequency region). If $\theta \in\left[0, \frac{1}{2}\right)$, we can apply Theorem 4.1 , to get that

$$
\|v(t)\| \lesssim\left\{\left\|v_{1}\right\|_{H^{-1} \cap L^{1}}+\left\|v_{0}\right\|_{L^{2} \cap L^{1}}\right\}(1+t)^{-\frac{1}{1-\theta}\left(\frac{n}{4}-\theta\right)},
$$

for all $n>4 \theta$;

$$
\begin{gathered}
\left\|v_{t}(t)\right\| \lesssim\left\{\begin{array}{l}
\left\{\left\|v_{1}\right\|_{L^{2} \cap L^{1}}+\left\|v_{0}\right\|_{H^{1} \cap L^{1}}\right\}(1+t)^{-\frac{1}{4 \theta},} \\
\left\{\left\|v_{1}\right\|_{L^{2} \cap L^{1}}+\left\|v_{0}\right\|_{H^{1} \cap L^{1}}\right\}(1+t)^{-\frac{1}{1-\theta}\left(\frac{n}{4}-\theta\right)-1}, \quad \text { if } n=1 \text { and } \theta \in\left[\frac{1}{4}, \frac{1}{2}\right),
\end{array}\right. \\
\|\nabla v(t)\| \lesssim\left\{\left\|v_{1}\right\|_{L^{2} \cap L^{1}}+\left\|v_{0}\right\|_{H^{1} \cap L^{1}}\right\}(1+t)^{-\frac{1}{1-\theta}\left(\frac{n}{4}+\frac{1}{2}-\theta\right)},
\end{gathered}
$$

for $n \geq 1$.

Now for $\theta \in\left[\frac{1}{2}, 1\right]$ we have $\alpha \leq 2 \theta$. Then, using Theorem 4.2 we can obtain the following estimate for the norm of solution, for $n \geq 3$ :

$$
\|v(t)\| \lesssim\left\{\left\|v_{1}\right\|_{H^{-1} \cap L^{1}}+\left\|v_{0}\right\|_{L^{2} \cap L^{1}}\right\}(1+t)^{-\frac{1}{\theta}\left(\frac{n}{4}-\frac{1}{2}\right)} ;
$$

and the following decay rates for the energy terms

$$
\left\|v_{t}(t)\right\|+\|\nabla v(t)\| \lesssim\left\{\left\|v_{1}\right\|_{L^{2} \cap L^{1}}+\left\|v_{0}\right\|_{H^{1} \cap L^{1}}\right\}(1+t)^{-\frac{n}{4 \theta},}
$$

in the case $n \geq 1$.

4.2.2 Plate equation with rotational inertia effects and fractional damping

In the previous works Charão et al. (2013a); D'Abbicco et al. (2016) for the plate equation with rotational inertia effects and fractional damping the authors 
considered the hypothesis $\theta \in[0,1]$. In this application we generalize this interval assuming that $\theta \in[0,2]$. Note that $\alpha>2 \theta$ if $\theta \in[0,1)$ and $\alpha \leq 2 \theta$ if $\theta \in[1,2]$, that is, to obtain the results it will be necessary to separate in two cases. This situation is similar to that one for the wave equation with fractional damping when the interval $\theta \in[0,1]$ is separated at intervals $\theta \in\left[0, \frac{1}{2}\right)$ and $\theta \in\left[\frac{1}{2}, 1\right]$ (see Charão et al. (2013b); Ikehata and Natsume (2012); D'Abbicco and Reissig (2014)).

We consider the following problem:

$$
\left\{\begin{array}{c}
v_{t t}(t, x)-\Delta v_{t t}(t, x)+\Delta^{2} v(t, x)+(-\Delta)^{\theta} v_{t}(t, x)=0, \quad t \geq 0, \quad x \in \mathbb{R}^{n} \\
v(0, x)=v_{0}(x), \quad v_{t}(0, x)=v_{1}(x)
\end{array}\right.
$$

with $\theta \in[0,2]$. That is, we take $\delta=1$ and $\alpha=2$ in (1)-(2).

The associated energy is given by

$$
E_{v}(t)=\frac{1}{2}\left\{\left\|v_{\mathrm{t}}(t)\right\|^{2}+\left\|\nabla v_{t}(t)\right\|^{2}+\|\Delta v(t)\|^{2}\right\}
$$

If $\theta \in[0,1)$, we consider $\left|\gamma_{1}\right|=0$ and $\frac{1}{2 \beta}=\frac{1}{2-\theta}\left(\frac{n}{4}-\theta\right)$. By item ( $i$ ) from Theorem 4.1, we have the following estimate for the $L^{2}$-norm of solution, for all $n>4 \theta$ :

$$
\|v(t)\| \lesssim\left\{\left\|v_{1}\right\|_{H^{r} \cap L^{1}}+\left\|v_{0}\right\|_{H^{s} \cap L^{1}}\right\}(1+t)^{-\frac{1}{2-\theta}\left(\frac{n}{4}-\theta\right)},
$$

with $r=\frac{(1-\theta)(n-4 \theta)}{2(2-\theta)}-1$ and $s=\frac{(1-\theta)(n-4 \theta)}{2(2-\theta)}$.

In order to get decay rates for the energy, we estimate each term of it separately. In the next three estimates we assume $\theta \in[0,1)$. At item (ii) of Theorem 4.1 we can choose $\left|\gamma_{2}\right|=0$ with $\frac{1}{2 \beta}=\frac{n}{4 \theta}$ in (25) and $\frac{1}{2 \beta}=\frac{1}{2-\theta}\left(\frac{n}{4}-\theta\right)+1$ in (26), to obtain

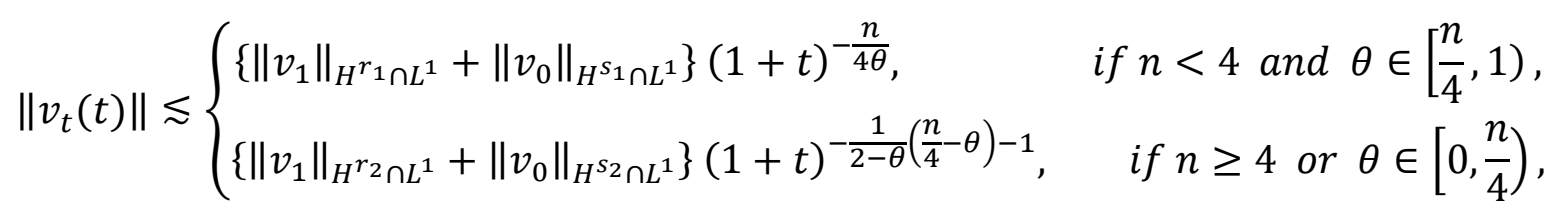


with $r_{1}=\frac{(1-\theta) n}{2 \theta}, s_{1}=\frac{(1-\theta) n}{2 \theta}+1, r_{2}=\frac{(1-\theta)(n-8 \theta+8)}{2(2-\theta)}$ and $s_{2}=\frac{(1-\theta)(n-8 \theta+8)}{2(2-\theta)}+1$.

Now, if $\left|\gamma_{2}\right|=1$, with $\frac{1}{2 \beta}=\frac{1}{\theta}\left(\frac{n}{4}+\frac{1}{2}\right)$ in (25) and $\frac{1}{2 \beta}=\frac{1}{2-\theta}\left(\frac{n}{4}+\frac{1}{2}-\theta\right)+1$ in (26), by item (ii) in Theorem 4.1 it follows that

$$
\left\|\nabla v_{t}(t)\right\| \lesssim \begin{cases}\left\{\left\|v_{1}\right\|_{H^{p} \cap L^{1}}+\left\|v_{0}\right\|_{H^{q} \cap L^{1}}\right\}(1+t)^{-\frac{3}{4 \theta},} & \text { if } n=1 \text { and } \theta \in\left[\frac{3}{4}, 1\right), \\ \left\{\left\|v_{1}\right\|_{H^{r} \cap L^{1}}+\left\|v_{0}\right\|_{H^{s} \cap L^{1}}\right\}(1+t)^{-\frac{1}{2-\theta}\left(\frac{n}{4}+\frac{1}{2}-\theta\right)-1}, & \text { if } n \geq 2 \text { or } \theta \in\left[0, \frac{3}{4}\right),\end{cases}
$$

where $p=1+\frac{(1-\theta)(n+2)}{2 \theta}, q=2+\frac{(1-\theta)(n+2)}{2 \theta}, r=1+\frac{(1-\theta)(n-8 \theta+10)}{2(2-\theta)}$ and $s=2+\frac{(1-\theta)(n-8 \theta+10)}{2(2-\theta)}$.

On the other hand, if $\left|\gamma_{1}\right|=2$ and $\frac{1}{2 \beta}=\frac{1}{2-\theta}\left(\frac{n}{4}+1-\theta\right)$, the Theorem 4.1, item (i), gives us:

$$
\|\Delta v(t)\| \lesssim\left\{\left\|v_{1}\right\|_{H^{r} \cap L^{1}}+\left\|v_{0}\right\|_{H^{s} \cap L^{1}}\right\}(1+t)^{-\frac{1}{2-\theta}\left(\frac{n}{4}+1-\theta\right)}
$$

where $r=1+\frac{(1-\theta)(n-4 \theta+4)}{2(2-\theta)}$ and $s=2+\frac{(1-\theta)(n-4 \theta+4)}{2(2-\theta)}$.

The result for the case $\theta \in[1,2]$ is obtained similarly to the previous case, using the Theorem 4.2 instead of Theorem 4.1. The decay rates obtained to the $L^{2}$-norm of solution are:

$$
\|v(t)\| \lesssim\left\{\left\|v_{1}\right\|_{H^{-1} \cap L^{1}}+\left\|v_{0}\right\|_{L^{2} \cap L^{1}}\right\}(1+t)^{-\frac{1}{\theta}\left(\frac{n}{4}-1\right)}
$$

for $n>4$. For the energy terms we have:

$$
\begin{gathered}
\left\|v_{t}(t)\right\| \lesssim\left\{\left\|v_{1}\right\|_{L^{2} \cap L^{1}}+\left\|v_{0}\right\|_{H^{1} \cap L^{1}}\right\}(1+t)^{-\frac{n}{4 \theta}} \\
\left\|\nabla v_{t}(t)\right\| \lesssim\left\{\left\|v_{1}\right\|_{H^{1} \cap L^{1}}+\left\|v_{0}\right\|_{H^{2} \cap L^{1}}\right\}(1+t)^{-\frac{1}{\theta}\left(\frac{n}{4}+\frac{1}{2}\right)} \\
\|\Delta v(t)\| \lesssim\left\{\left\|v_{1}\right\|_{H^{1} \cap L^{1}}+\left\|v_{0}\right\|_{H^{2} \cap L^{1}}\right\}(1+t)^{-\frac{n}{4 \theta}}
\end{gathered}
$$

Remark 4.1. The plate equation without the inertia rotational effects, that is, (1)-(2) with $\alpha=2$ and $\delta=0$, does not have the structure of regularity-loss. In 
this case, the decay rates are equal to the above rates, but without assume additional regularity at the initial data.

\subsubsection{Boussinesq equation with fractional damping}

In this subsection, we want to show that it is possible to add terms of type $(-\Delta)^{\delta_{1}} v_{t t},(-\Delta)^{\theta_{1}} v_{t},(-\Delta)^{\alpha_{1}} v$ in the equation (1) in order to obtain decay rates for the total energy and for the $L^{2}$-norm of solution from the previous results. However, we consider just a simple example to illustrate this case.

In Polat and Ertas (2009) the authors studied the Boussinesq equation (IBq) with the strong dissipation $\Delta v_{t}$ (see Wang and Xu $(2013,2012)$ ). Next, we regard a more general case than the linear problem studied in Polat and Ertas (2009), considering the following IBq with fractional damping in $\mathbb{R}^{n}$ :

$v_{t t}(t, x)-\Delta v(t, x)-\Delta v_{t t}(t, x)+\Delta^{2} v(t, x)+(-\Delta)^{\theta} v_{t}(t, x)=0, t \geq 0, x \in \mathbb{R}^{n}$

with $\theta \in[0,1]$ and initial data

$$
v(0, x)=v_{0}(x), v_{t}(0, x)=v_{1}(x)
$$

The energy associated to this equation is given by

$$
E_{v}(t)=\frac{1}{2}\left\{\left\|v_{t}(t)\right\|^{2}+\|\nabla v(t)\|^{2}+\left\|\nabla v_{t}(t)\right\|^{2}+\|\Delta v(t)\|^{2}\right\}
$$

Applying the Fourier transform in (27) and (28), we obtain

$$
\left\{\begin{array}{c}
\left(1+|\xi|^{2}\right) \hat{v}_{t t}(t, \xi)+|\xi|^{2 \theta} \hat{v}_{t}(t, \xi)+\left(|\xi|^{2}+|\xi|^{4}\right) \hat{v}(t, \xi)=0 \\
\hat{v}(0, \xi)=\hat{v}_{0}(\xi), \quad \hat{v}_{t}(0, \xi)=\hat{v}_{1}(\xi)
\end{array}\right.
$$

This problem is not a specific case of the initial problem (4). But we note that for $|\xi|<\varepsilon$ is valid the equivalence

$$
|\xi|^{2} \approx|\xi|^{2}+|\xi|^{4}
$$


which leads us to conclude that the estimates in the low frequency for this equation are the same of Propositions 2.1 and 2.2 considering $\alpha=\delta=1$.

On the other hand, if $|\xi| \geq \varepsilon$, then

$$
|\xi|^{4} \approx|\xi|^{2}+|\xi|^{4}
$$

and therefore the estimates and regularity in the initial data for this equation at high frequency are given by Proposition 3.1, with $\alpha=2$ and $\delta=1$. This gives us the following results:

Theorem 4.3. If $\theta \in\left[0, \frac{1}{2}\right)$ and $\left(v_{0}, v_{1}\right) \in\left[H^{s}\left(\mathbb{R}^{n}\right) \cap L^{1}\left(\mathbb{R}^{n}\right)\right] \times\left[H^{r}\left(\mathbb{R}^{n}\right) \cap L^{1}\left(\mathbb{R}^{n}\right)\right]$, with $r$ and s specified in each case below, then the solution of the problem (27)(28) satisfies the following decay estimates:

(i) If $n>4 \theta$ then for $t \geq 0$

$$
\|v(t)\| \lesssim\left\{\left\|v_{1}\right\|_{H^{r} \cap L^{1}}+\left\|v_{0}\right\|_{H^{s} \cap L^{1}}\right\}(1+t)^{-\frac{1}{1-\theta}\left(\frac{n}{4}-\theta\right)},
$$

with $r=\frac{n-4 \theta}{2}-1$ and $s=\frac{n-4 \theta}{2}$.

(ii) If $n \geq 1$ then for $t \geq 0$

$$
\|\nabla v(t)\| \lesssim\left\{\left\|v_{1}\right\|_{H^{r} \cap L^{1}}+\left\|v_{0}\right\|_{H^{s} \cap L^{1}}\right\}(1+t)^{-\frac{1}{1-\theta}\left(\frac{n}{4}+\frac{1}{2}-\theta\right)},
$$

with $r=\frac{n+2-4 \theta}{2}$ and $s=1+\frac{n+2-4 \theta}{2}$;

$$
\|\Delta v(t)\| \lesssim\left\{\left\|v_{1}\right\|_{H^{r} \cap L^{1}}+\left\|v_{0}\right\|_{H^{s} \cap L^{1}}\right\}(1+t)^{-\frac{1}{1-\theta}\left(\frac{n}{4}+1-\theta\right)},
$$

with $r=1+\frac{n+4-4 \theta}{2}$ and $s=2+\frac{n+4-4 \theta}{2}$;

$$
\left\|v_{t}(t)\right\| \lesssim\left\{\begin{array}{l}
\left\{\left\|v_{1}\right\|_{H^{r_{1} \cap L^{1}}}+\left\|v_{0}\right\|_{H^{s_{1}} \cap L^{1}}\right\}(1+t)^{-\frac{1}{4 \theta},} \\
\left\{\left\|v_{1}\right\|_{H^{r_{2} \cap L^{1}}}+\left\|v_{0}\right\|_{H^{s_{2}} \cap L^{1}}\right\}(1+t)^{-\frac{1}{1-\theta}\left(\frac{n}{4}-\theta\right)-1}, \quad \text { if } n=1 \text { and } \theta \epsilon\left[\frac{1}{4}, \frac{1}{2}\right),
\end{array}\right.
$$

with $r_{1}=\frac{(1-\theta) n}{2 \theta}, s_{1}=1+\frac{(1-\theta) n}{2 \theta}, r_{2}=\frac{n-8 \theta+4}{2}$ and $s_{2}=1+\frac{n-8 \theta+4}{2}$; 


$$
\left\|\nabla v_{t}(t)\right\| \lesssim\left\{\left\|v_{1}\right\|_{H^{r} \cap L^{1}}+\left\|v_{0}\right\|_{H^{s} \cap L^{1}}\right\}(1+t)^{-\frac{1}{1-\theta}\left(\frac{n}{4}+\frac{1}{2}-\theta\right)-1},
$$

with $r=1+\frac{n-8 \theta+6}{2}$ and $s=2+\frac{n-8 \theta+6}{2}$.

Theorem 4.4. If $\theta \epsilon\left[\frac{1}{2}, 1\right]$ and $\left(v_{0}, v_{1}\right) \epsilon\left[H^{s}\left(\mathbb{R}^{n}\right) \cap L^{1}\left(\mathbb{R}^{n}\right)\right] \times\left[H^{r}\left(\mathbb{R}^{n}\right) \cap L^{1}\left(\mathbb{R}^{n}\right)\right]$, with $r$ and $s$ defined in each case, the following decay estimates for the problem (27)-(28) are true:

(i) If $n>2$ then for $t \geq 0$

$$
\|v(t)\| \lesssim\left\{\left\|v_{1}\right\|_{H^{r} \cap L^{1}}+\left\|v_{0}\right\|_{H^{s} \cap L^{1}}\right\}(1+t)^{-\frac{1}{\theta}\left(\frac{n}{4}-\frac{1}{2}\right)},
$$

with $r=-1$ and $s=0$ in case $\theta=1$, and with $r=\frac{(1-\theta)(n-2)}{2 \theta}-1$ and $s=\frac{(1-\theta)(n-2)}{2 \theta}$ in case $\theta<1$.

(ii) If $n \geq 1$ then for $t \geq 0$

$$
\|\nabla v(t)\|+\left\|v_{t}(t)\right\| \lesssim\left\{\left\|v_{1}\right\|_{H^{r} \cap L^{1}}+\left\|v_{0}\right\|_{H^{s} \cap L^{1}}\right\}(1+t)^{-\frac{n}{4 \theta}}
$$

with $r=0$ and $s=1$ in case $\theta=1$, and with $r=\frac{(1-\theta) n}{2 \theta}$ and $s=1+\frac{(1-\theta) n}{2 \theta}$ in case $\theta<1$;

$$
\|\Delta v(t)\|+\left\|\nabla v_{t}(t)\right\| \lesssim\left\{\left\|v_{1}\right\|_{H^{r} \cap L^{1}}+\left\|v_{0}\right\|_{H^{s} \cap L^{1}}\right\}(1+t)^{-\frac{1}{\theta}\left(\frac{n}{4}+\frac{1}{2}\right)},
$$

with $r=1$ and $s=2$ in case $\theta=1$, and with $r=1+\frac{(1-\theta)(n+2)}{2 \theta}$ and $s=2+\frac{(1-\theta)(n+2)}{2 \theta}$ at case $\theta<1$.

\section{ACKNOWLEDGMENTS}

The second author has been partially supported by Conselho Nacional de Desenvolvimento Científico e Tecnológico - CNPq, Proc. 308868/2015-3 and 314398/2018-0. In addition, the authors would like to thank the reviewer for his or her valuable time and useful contributions. 


\section{REFERENCES}

Biler, P., Karch, G., Woyczy'nski, W. (2001). Asymptotics for conservation laws involving Lévy diffusion generators. Studia Mathematica, 148(2), 171-192.

Charão, R. C., da Luz, C. R., Ikehata, R. (2013a). New decay rates for a problem of plate dynamics with fractional damping. Journal of Hyperbolic Differential Equations, 10(3), 563-575.

Charão, R. C., da Luz, C. R., Ikehata, R. (2013b). Sharp decay rates for wave equations with a fractional damping via new method in the Fourier space. Journal of Mathematical Analysis and Applications, 408(1), 247-255.

Charão, R. C., Espinoza, J. C. T., Ikehata, R. (2020). A second order fractional differential equation under effects of a super damping. Commun Pure Appl Anal, 19(9), 4433-4454.

Córdoba, A., Córdoba, D. (2004). A maximum principle applied to quasi-geostrophic equations. Communications in Mathematical Physics, 249, 511-528.

D'Abbicco, M., Ebert, M. R. (2014). Diffusion phenomena for the wave equation with structural damping in the $I^{p}-I^{q}$ framework. Journal of Differential Equations, 256(7), 2307-2336.

D'Abbicco, M., Reissig, M. (2014). Semilinear structural damped waves. Mathematical Methods in the Applied Sciences, 37(11), 1570-1592.

D'Abbicco, M., Charão, R. C., da Luz, C. R. (2016). Sharp time decay rates on a hyperbolic plate model under effects of an intermediate damping with a time-dependent coefficient. Discrete and Continuous Dynamical Systems Series A, 36, 2419-2447.

Dharmawardane, P. M. N., Rivera, J. E. M., Kawashima, S. (2010). Decay property for second order hyperbolic systems of viscoelastic materials. Journal of Mathematical Analysis and Applications, 366, 621-635.

Fino, A., Karch, G. (2010). Decay of mass for nonlinear equation with fractional laplacian. Monatshefte für Mathematik, 160(4), 375-384.

Gauer Palma, M. F. (2013). Método da energia no espaço de Fourier para equações de evolução em $\mathbb{R}^{n}$ com dissipação fracionária. Masters dissertation, Department of Mathematics, Federal University of Santa Catarina, Brazil.

Ide, K., Kawashima, S. (2008). Decay property of regularity-loss type and nonlinear effects for dissipative Timoshenko system. Mathematical Models and Methods in Applied Sciences, 18(7), 1001-1025.

Ikehata, R., Natsume, M. (2012). Energy decay estimates for wave equations with a fractional damping. Differential Integral Equations, 25(9-10), 939-956. 
Ikehata, R., Todorova, G., Yordanov, B. (2013). Wave equations with strong damping in Hilbert spaces. Journal of Differential Equations, 254(8), 3352-3368.

Karch, G. (2000). Selfsimilar profiles in large time asymptotics of solutions to damped wave equations. Studia Mathematica, 143(2), 175-197.

Komornik, V. (1996). Differential and integral inequalities. Univ Beograd Publ Elektrotehn Fak Ser Math, (7), 55-67.

Kubo, T., Kawashima, S. (2009). Decay property of regularity-loss type and nonlinear effects for some hyperbolic-elliptic system. Kyushu Journal of Mathematics, 63(1), 139-159.

Lu, X., Reissig, M. (2009). Rates of decay for structural damped models with decreasing in time coefficients. International Journal of Dynamical Systems and Differential Equations, 2(1-2), 2155.

da Luz, C. R., Ikehata, R., Charão, R. C. (2015). Asymptotic behavior for abstract evolution differential equations of second order. Journal of Differential Equations, (259), 5017-5039.

Polat, N., Ertas, A. (2009). Existence and blow-up of solution of Cauchy problem for the generalized damped multidimensional boussinesq equation. Journal of Mathematical Analysis and Applications, 349(1), 10-20.

Sugitani, Y., Kawashima, S. (2010). Decay estimates of solutions to a semi-linear dissipative plate equation. Journal of Hyperbolic Differential Equations, 7(3), 471-501.

Umeda, T., Kawashima, S., Shizuta, Y. (1984). On the decay of solutions to the linearized equations of electro-magneto-fluid dynamics. Japan Journal of Applied Mathematics, 1(2), 435-457.

Vázquez, J. L. (2010). Nonlinear diffusion with fractional laplacian operators. Nonlinear Partial Differential Equations, pp. 271-298.

Wang, S., Xu, H. (2012). On the asymptotic behavior of solution for the generalized IBq equation with hydrodynamical damped term. Journal of Differential Equations, 252(7), 42434258.

Wang, S., Xu, H. (2013). On the asymptotic behavior of solution for the generalized IBq equation with Stokes damped term. Zeitschrift für angewandte Mathematik und Physik ZAMP, 64(3), 719-731.

Wirth, J. (2006). Wave equations with time-dependent dissipation. I. non-effective dissipation. Journal of Differential Equations, 222, 487-514. 


\section{Authorship contributions}

\section{1 - Cleverson Roberto da Luz}

Docente do Departamento de Matemática na Universidade Federal de Santa Catarina https://orcid.org/0000-0002-5972-5677 - cleverson.luz@ufsc.br

Contribuição: revisão bibliográfica, obtenção de resultados, análise dos resultados e revisão do artigo.

\section{2 - Maíra Fernandes Gauer Palma}

Docente do Departamento de Matemática na Universidade Federal de Santa Catarina https://orcid.org/0000-0003-1611-390X-maira.gauer@ufsc.br

Contribuição: revisão bibliográfica, obtenção de resultados, análise dos resultados e revisão do artigo. 